\title{
Comprehensively Addressing Postpartum Maternal Health: a Content and Image Review of Commercially Available Mobile Health Apps
}

Laura Tucker ( $\nabla$ lat98@pitt.edu )

University of Pittsburgh School of Medicine https://orcid.org/0000-0002-2998-5590

Alan Cuevas Villagomez

University of Pittsburgh School of Medicine

Tamar Krishnamurti

University of Pittsburgh School of Medicine

\section{Research article}

Keywords: Pregnancy, postpartum, peripartum, maternal health, mHealth, mobile health, maternal morbidity, health education, health disparity, mobile apps

Posted Date: August 10th, 2020

DOl: https://doi.org/10.21203/rs.3.rs-49594/v1

License: (c) (i) This work is licensed under a Creative Commons Attribution 4.0 International License. Read Full License

Version of Record: A version of this preprint was published at BMC Pregnancy and Childbirth on April 20th, 2021. See the published version at https://doi.org/10.1186/s12884-021-03785-7. 


\section{Abstract}

Background: The United States is currently facing a maternal morbidity and mortality crisis, with the highest rates of any resource-rich nation. In efforts to address this, new guidelines for postpartum care suggest that mobile health apps can help provide complementary clinical support for new mothers during the postpartum period. However, to date no study has evaluated existing mobile health tools targeted to this time period for sufficiency of maternal health information, inclusivity of people of color, or accessibility to users.

Methods: Preferred Reporting Items for Systematic Reviews and Meta-Analyses (PRISMA) standards were used to review the peripartum apps from the Apple and Google Play stores in either the Health/Fitness, Medical, or Education categories. Apps were then evaluated for extent and quality of maternal health information, inclusivity of people of color, and accessibility to app users.

Results: Of the 301 apps from the Apple and Google Play stores, 25 met criteria for final evaluation. Of the 30 maternal health topics coded for, the median number addressed by apps was 19.5 (65\%). Peripartum behaviors were more frequently addressed than peripartum outpatient care topics and peripartum acute health risks. The amount of maternal health information correlated positively with the Mobile Application Rating Scale (MARS) quality score of the app, and inclusivity of people of color in app imagery also correlated positively with the MARS quality score. Only 8 apps (32\%) portrayed greater than $24 \%$ images of people of color- the percent of non-white Americans according to 2019 census estimates. There was no correlation between number of users as estimated by number of store ratings and MARS quality. In addition, apps with clinical authority had greater MARS engagement, information, aesthetics, and quality scores, but did not have greater numbers of store ratings.

Conclusions: Current commercially available peripartum apps overall do not provide adequate maternal health information, are not inclusive of women of color, and are not optimally accessible to the target users. Apps authored with clinical authority and higher-quality apps, by MARS score, are more likely to meet these standards, but are not more likely to be downloaded and used.

\section{Background}

The United States is currently facing a maternal morbidity and mortality crisis. The maternal mortality rate has more than doubled since 1990, and currently stands at 17.4 deaths per 100,000 live births-- the highest of any resource-rich nation [1]. The leading documented causes of maternal death are infection, hemorrhage, cardiomyopathy, other cardiovascular conditions, pulmonary embolism, stroke, hypertensive disorders, and amniotic fluid embolism [2]. Newly emerging data also suggests that psychosocial risks, such as intimate partner violence (IPV) and depression-induced suicide, may also play a key role in maternal mortality and morbidity occurring up to a year postpartum [3,4]. Furthermore, an additional 60,000 U.S. women experience severe maternal morbidity each year [5]. Pregnancy-related maternal mortality and morbidity is disproportionately high among women of color whereby non-Hispanic Black 
women are more than 3 times more likely to experience pregnancy-related death than non-Hispanic white women [6-8]. There are also disparities in cause of death, with Black women being more likely to suffer from pregnancy-related cardiomyopathy, pulmonary embolism, and hypertensive disorders [9]. In the peripartum period, Black and Hispanic women are at greater risk than white women of both IPV and depression $[10,11,12]$.

Peripartum health risks can be acute or chronic [13], and confounding symptoms with traditional postpartum experiences can lead to lack of recognition of more severe health risks. For example, while fatigue is a common consequence of postpartum sleep disturbance [14], it may also be an indicator of postpartum depression [15] or cardiomyopathy [16]. A 2018 nine-state maternal mortality review committee demonstrated that an overall lack of knowledge about warning signs and when to receive medical help was one of the most common factors contributing to postpartum maternal mortality [17]. In attempts to more comprehensively reach patients during the postpartum period, guidelines from the American College of Obstetrics and Gynecology (ACOG) have been released suggesting that mobile health apps can help provide supplementary clinical support [18].

Commercial apps communicating about pregnancy-related health, specifically, are becoming increasingly common $[19,20]$, with healthcare providers endorsing that they play a growing role in maternity care [18, 19]. Using mobile health apps as a widespread form of supplementary clinical support shows promise in both increasing patient education and in narrowing the knowledge gap in health disparate communities $[21,22]$. Most women of reproductive age own smartphones, even among the lowest income bracket. Moreover, within this group there is an almost equal distribution across Black, White, and Latino populations [23]. Approximately $80 \%$ of smartphone owners use their phones to access health information, and Black and Latino smartphone owners are more likely than White smartphone owners to use their phones to research health conditions $[24,25]$. To date, however, there is no existing evaluation of how well pregnancy apps explicitly address maternal mortality or morbidity risks or whether the requisite information is presented in a way that makes it accessible to those most at risk.

Even when apps are adequately addressing critical public health information, their use can be impeded by a failure to tailor them to the specific needs of their audience $[26,27]$. Due to the striking maternal health disparities which currently exist, the risk of further distancing health disparate communities has worrying consequences. Several studies have critically reviewed the content of pregnancy apps $[20,28,29]$, with Thomas \& Lupton (2016) finding that pregnancy and other reproductive health apps fail to be inclusive of diverse pregnant people. These kinds of findings raise concerns of bias in the language and imagery used in pregnancy-health apps. In the context of smartphone apps addressing pregnancy-related health risks, meeting the needs of the intended audience requires that such apps be specifically inclusive of health disparate groups. In this analysis, established methods [30-32] of app review were drawn upon to scope the content of commercially available apps for their ability to adequately inform users about behavioral, outpatient, and acute peripartum risk factors. This was done by determining whether they include the requisite maternal health information to address the leading causes of maternal morbidity and mortality as well as other common postpartum risk factors. Each app was then reviewed for 
inclusivity in language and imagery and for accessibility to understand whether apps that fill a critical pregnancy-related health information gap are also inclusive of health disparate communities.

\section{Methods}

\section{Framework}

This study employed the Preferred Reporting Items for Systematic Reviews and Meta-Analyses (PRISMA) standards [33], following similar implementation to other mHealth app reviews of content [30-32].

\section{Search Strategy}

The search was limited to the Apple App Store and Google Play. To comprehensively capture mobile health apps addressing both the pregnancy and postpartum period, a systematic search was conducted using a combination of key terms, reviewed by both obstetric and family medicine providers. These key terms included:

- Pregnancy

- Fetal development

- Fetal growth

- Labor and Delivery

- Postpartum

- Maternal/maternity

The search was limited to apps in the Health/Fitness, Medical, and Education categories (as opposed to, e.g. "entertainment") to identify those that may contain clinically relevant content.

\section{Selection Criteria}

The review was limited to the top 200 apps identified from each search term applied. Apps that met inclusion criteria and were available in both stores were initially evaluated to identify substantial differences in content and imagery across operating systems. If the app showed consistency, the most recently updated app was included in the review.

\section{Inclusion Criteria}

Currently available apps (as of August 2019) targeting pregnant patients or containing content for those who have delivered within the preceding three months (those in the " 4 th trimester") were eligible for review. Apps were required to address education or provide support for physical or mental health needs. For example, apps could be marketed for education, health tracking, medical appointment reminders, or managing stress and anxiety specifically related to pregnancy, childbirth, or postpartum. To meet those specifications, the inclusion criteria were as follows: (1) apps targeted to pregnant or postpartum women 
(up to 3 months postpartum); (2) apps whose primary content was focused on the mother (i.e. not primarily child development or parenting) (2) available through Apple App Store or Google Play; (3) English language; (4) free or paid apps costing less than $\$ 10$ per app (5) apps available in the following Apple App Store categories: Health \& Fitness, and Medical; and (6) apps available in the following Google Play categories: Education, Health \& Fitness, Medical.

\section{Exclusion Criteria}

Apps were excluded if they were (1) general parenting apps (2) intended for health care professionals; (3) targeted explicitly towards men or partners of pregnant people (4) classified as e-books by app store description or reviewers (5) entertainment or social networking apps or (6) targeting a single specific symptom or condition that might be experienced in the postpartum period (i.e. cognitive behavioral therapy apps for depression).

\section{Screening Process}

After removal of duplicates, the title and store descriptions of all apps identified in an initial search were screened to determine eligibility for full review. Apps that were eligible for full review were downloaded. For the full review, one primary reviewer evaluated the entire set of Android and iOS apps. The apps were then evaluated independently by one of three secondary reviewers, who were randomly assigned a subset of apps to review. If any uncertainties or disagreements were identified in coding, they were reviewed by the entire research team and resolution was achieved by group consensus.

\section{Measures}

\section{Maternal Health Information}

To comprehensively capture information that may be considered necessary for a peripartum app to serve as a clinical support tool, an a priori coding scheme for the apps' maternal health content was developed based on the primary causes of severe maternal morbidity and mortality and referencing ACOG and CDC guidelines for postpartum care topics [18,34]. Apps were coded for the presence (or absence) of specific maternal health content and for whether that information was connected to a citation from a professional medical organization or peer-reviewed scientific literature, which was labeled "clinical authority." Qualitative notes were made of inaccuracies in health information. Table 1 provides details on the 30 specific codes for maternal health information. Elements were separated into three categories: Peripartum Behaviors, Peripartum Outpatient Care, and Peripartum Acute Health Risks.

Table 1. Coding scheme for maternal clinical risk information.

Inclusivity

To evaluate inclusivity of the apps, each was coded for references to race in the written content and imagery embedded in the app. All references were coded for the presence or absence of race-based 
stereotyping and the specific instance was documented with an open-ended note. A second rater then reviewed the instance and note and created a binary code for presence/absence of a biased reference. Definitions for all coding can be found in Supplemental Appendix A. To categorize an app as offering racial inclusivity, it was noted whether at least $24 \%$ of app-embedded images displayed non-white presenting individuals. This threshold was chosen to reflect the percentage of the U.S. identifying as a race other than white according to the 2019 US Census estimate [35].

\section{Accessibility}

The Mobile Application Rating Scale (MARS) was used to assess the quality of mobile apps. This scale consists of 4 subscales: engagement, functionality, aesthetics, and information quality. These subscales are averaged to produce a final mean quality score. Two optional separate subscales are provided to assess subjective quality and perceived impact of the app on user knowledge and behaviors. Each item considered within a subscale uses a 5 -point Likert scale ( $1=$ Inadequate, $2=$ Poor, $3=A c c e p t a b l e, ~ 4=$ Good, $5=$ Excellent). MARS scores from only the primary rater were used, as this scale has not been validated to incorporate ratings from multiple users in the same score [36].

To assess relative rates of usage in the general public, the total number of Apple and/or Google Play Store ratings for each app was recorded.

\section{Data Analysis}

Descriptive statistics, frequencies, correlations, and independent $t$ tests were used to summarize the search results and evaluation scores assigned to each app. Data analysis was conducted using IBM SPSS 24.0.

\section{Results}

The PRISMA flowchart of the search process can be seen in Figure 1.

Figure 1. Preferred Reporting Items for Systematic Reviews and Meta-Analyses flowchart of search process for educational mHealth apps with postpartum maternal morbidity and mortality risk content

\section{Maternal Health Content}

Of the 25 applications coded, only one, "What to Expect" addressed each of the 30 medical topics included in the Maternal Health coding scheme. The median number of elements addressed by each app was $19.5(65 \%)$, with a range from $4(13.3 \%)$ to $30(100 \%)$. Apps affiliated with a medical organization (i.e. "Circle by Joseph Health" and "Circle by Swedish") addressed greater than $90 \%$ of the peripartum health topics, whereas the remaining, commercially developed, apps almost universally fell below this threshold. The exceptions to this were the commercially developed "WebMD Pregnancy" and "The Bump," which addressed $29(96.7 \%)$ and 28 (93.3\%) of the topics, respectively. A significant $(r(23)=0.643$, $p=0.001)$ positive correlation was found between the percent of elements addressed in an app and the 
app's MARS quality score (Figure 2). Ten of the 25 apps reviewed (40\%) met the threshold for clinical authority. Examples of information addressing a maternal health topic without clinical authority include offering guidance on postpartum depression by explaining that mothers who are "feeling down" are likely feeling so because of dissatisfaction with their bodies, suggesting they should "wear flats," and "join an online mom group to beat the postpartum blues."

Figure 2. MARS overall quality score vs percent of maternal health information addressed by app.

Peripartum behaviors were the most frequently addressed by apps, with $5 / 9(56 \%)$ addressed by more than $75 \%$ of apps in contrast to $4 / 12$ (33\%) of peripartum outpatient care topics and $0 / 9(0 \%)$ of peripartum acute health risks. On average, peripartum behaviors were addressed by $70 \%$ of apps, peripartum outpatient care by $59 \%$, and peripartum health risks by $45 \%$. The most widely addressed were breastfeeding/breast health and depression, which were each discussed in 23 apps (92\%) (Figure 3 ).

Figure 3. Percent of mobile applications addressing elements of peripartum behaviors, peripartum outpatient care, and peripartum acute health risks.

\section{Inclusivity}

Only $32 \%$ (8/24 apps; one app did not contain images) of applications portrayed greater than $24 \%$ of people of color in their imagery. Positive associations were found between the percent of images including people of color and both the MARS quality score $(r(22)=0.535, p=0.007)$ and the percent of medical topics addressed $(r(23)=0.756, p<0.001)$. No significant correlation was found between the number of ratings and percent of people of color $(r(23)=0.271, p=0.20)$. Of the top-10 most-rated apps, only four ("Pregnancy Tracker-BabyCenter," "What to Expect," "The Bump," and "Ovia Pregnancy Tracker") reached the $24 \%$ diversity threshold. No race-biased language was noted in any of the apps, but 5 apps $(20 \%)$ were noted to have race-biased imagery in the form of depicting women of color exclusively as patients while white individuals were depicted in diverse roles or in majority healthcare worker roles.

\section{Accessibility}

The average overall MARS score (quality mean score) for the 25 apps ranged from 1.9 to 4.7 , with a mean quality score of 3.7, representing an "Acceptable" rating. Overall, apps scored high on functionality (mean=4.3, SD=0.5) and lower in engagement (mean=3.4, $S D=1.1$ ), aesthetics (mean=3.4, SD=1.1), information (mean $=3.5, S D=0.9$ ), and overall quality (mean=3.7, $S D=0.7$ ). The lowest-scoring MARS subscale was the optional "Subjective Quality," which is not included in the overall quality score but takes into account how frequently a user might access the app if relevant to them, whether they would pay for the app, and whether they would recommend it to other users. Subjective quality ratings ranged widely from 1.0-5.0, (mean=3.0, SD=1.1). "Circle by Swedish" received the highest overall quality score, 4.7 ("Good"), followed by "What to Expect," "Pregnancy Tracker-BabyCenter," "WebMD Pregnancy," and "Track Pregnancy\&Baby:Preglife," each with a rating of 4.5 ("Good"). None of the apps received a quality score of "Excellent." 
No significant correlation was found between the applications' number of store ratings (a proxy for prevalence and popularity) and the quality of the application, as measured by both the proportion of the medical topics the app addressed $(r(23)=0.252, p=0.224)$ and by the MARS quality score $(r(23)=0.255, p=0.219)$. While the most-rated app, "Pregnancy Tracker-BabyCenter" (993,267 total ratings), addressed $90.3 \%$ of medical topics and received a MARS quality score of 4.5 ("Good"), the next-most rated application, "Pregnancy +" (685,310 ratings) addressed only $48.39 \%$ and received a MARS quality score of only 3.5 ("Acceptable"). "Circle by Swedish," which addressed $96.7 \%$ of medical topics and received the highest MARS quality score of the apps in this review at 4.7 ("Good") only received 1,216 ratings.

Apps with clinical authority showed a significantly greater mean percent of maternal health information addressed in the app $(t(20)=4.02, p=0.001)$. These apps also had higher overall MARS quality scores, $(t(23)=4.74, p<0.001)$, with higher ratings on the engagement $(t(23)=4.06, p<0.001)$, aesthetics $(t(17)=3.10, p=0.007)$, and information sub-scores $(t(23)=5.09, p<0.001)$. A significant difference was not observed in the MARS functionality score between the two groups of apps $(t(21)=1.15, p=0.265)$. There was also no significant difference in the number of store ratings between the apps with and without clinical authority.

\section{Highest-Quality Apps}

Four apps met or exceeded all of the following criteria for an acceptable peripartum mHealth application: 1) Authored with clinical authority; 2 ) addressed greater than or equal to $90 \%$ of maternal health information; 3 ) imagery portrayed at least $24 \%$ people of color; 4 ) received a MARS score of at least 4 ("Good") in each subcategory of engagement, functionality, aesthetics, and information; 5) did not depict race-biased imagery or language. These apps were "What to Expect," "Pregnancy Tracker - BabyCenter," "Circle by Swedish," and "Circle by Joseph Health."

\section{Discussion}

Updated ACOG guidelines have promoted the use of mobile app-based support in postpartum care [18]. This review has shown that while some peripartum apps provide the medical information, inclusivity, and accessibility that is optimal for a successful clinical support tool, there is wide variability among the currently available products and many apps fail to meet these standards. The most frequently downloaded apps do not necessarily communicate the most peripartum health information and are not more likely to be authored with clinical authority. Lack of patient knowledge of warning signs is known to be a leading contributor to postpartum hemorrhage, cardiovascular disease, and cardiomyopathy- three of the leading causes of postpartum morbidity and mortality in the US [17]. The absence of any reference to these specific health risks in over half of the apps reviewed suggests that current offerings are not adequate to provide critical information to those who seek it.

Peripartum behavior and outpatient health topics were addressed more frequently than acute mortality and morbidity risks. For example, breastfeeding health information was provided by almost every app. 
This highlights an area where the current mobile apps can well serve postpartum women, the majority of whom have difficulty breastfeeding and for whom access to education provides measurable benefits [37, 38]. The content covered may be in response to market demand from app users, given that peripartum women have also expressed desire for additional education regarding postpartum depression, contraception, and physical well-being [39], each of which were addressed in the majority of the apps. However, the lack of clinical authority supporting a majority of the apps gives pause to recommending them for medical information, exemplified by the app whose mental health discussion only addresses only postpartum blues and recommends only online support groups. While social support online and in person can be protective for postpartum depression [40,41], there is a striking increased risk of mortality and morbidity for women who do not receive professional medical and psychological treatment [42]. Connecting patients with apps which minimize or trivialize mental health information may inadvertently put them at greater risk of negative consequences.

The apps also tended to lack inclusivity of women of color, and while more inclusive apps were associated with higher quality they were not more likely to be downloaded by users. One notable shortfall in inclusivity was seen in the rates of maternal health information provided by the apps. Infections and mental health, conditions that are more prevalent among non-Hispanic white women [17], were addressed by most of the apps, while embolism and cardiomyopathy, which disproportionately affect women of color, were infrequently addressed. A digital divide currently exists in the US, with black citizens less likely to have a desktop or laptop and more likely to prefer using mobile phones to seek health information [24]. As apps are a major form of information delivery on smartphones, the subtle bias in providing health information that disproportionately aids white women risks widening the health literacy and therefore peripartum mortality gap. Moreover, a majority of the peripartum apps were shown to lack inclusivity of women of color in their imagery. Black patients are more likely to prefer providers of the same race, [43, 44] and culturally appropriate information has been endorsed as a method to increase health equity in the reproductive health of black women [45]. The portion of apps which represent women of color only as patients also introduces potential damage, as increased racial minority representation in health professions has been shown to reduce health disparities [46]. Providers should be aware that using the currently-available commercial apps as clinical support tools may not be inclusive and effective for women of color.

Collectively, the apps received an overall only "Acceptable" MARS engagement, aesthetics, and information scores. Furthermore, there was no correlation between the quality of the app and the number of reviewers it had. Smartphone users are more likely to download an app they perceive as popular, associating popularity with quality, [47] and adoption and use of technology is directly related to its ease of use and perceived usefulness [48]. In addition, postpartum women report greatly reduced time for themselves and shifting priorities to competing life responsibilities above their own health [49]. These factors all may cause postpartum mothers to make less-researched decisions on which app to download, and to require high app engagement and quality to benefit mothers in their reduced free time. Access to personalized, evidence-based medicine via a mobile app has been shown to improve health behaviors of peripartum women. [50] However, while mobile apps offer a promising opportunity to improve postpartum 
care, the current commercially available options are not yet optimal for this purpose. Providers should be aware of the flaws in health information quality, inclusivity of women of color, and app accessibility when discussing app use with patients.

\section{Limitations}

In limiting this review to commercially available products, mHealth apps that have been developed specifically by health systems for their patient populations were unable to be included. While these types of apps will likely have higher degrees of clinical authority and capture postpartum health risks more comprehensively, they may also have more limited reach. Second, the intent of these apps may not explicitly be to serve as a clinical support tool, though this study surveys whether these tools could fit this purpose. The intent of women using these tools and whether they think of them as clinical support is also not known, and merits exploration in future work. A third limitation to this study, and all studies evaluating diversity of images, is the risk of mis-categorizing images of Black and other women of color. Having multiple reviewers make these judgments aimed to minimize this risk; however, it cannot be certain that the researchers' categorization is consistent with how the individual in the image would selfidentify. Similar limitations apply to discriminatory text and language. While a subjective measure, the evaluation by multiple reviewers and research group discussion of language attempted to mitigate these limitations. Finally, while the MARS scale is an accepted and validated measure, it was employed only by the researchers and not by the population using the app and, as such, the research team's judgments may not fully reflect those of the population.

\section{Conclusion}

While ACOG has recommended that mobile health apps can provide clinical support to patients during the postpartum period, no comprehensive review has before been done of the information, inclusivity, and accessibility of the currently available products. This review has found that many popular commercially available peripartum apps do not provide adequate maternal health information, are not inclusive of women of color, and are not optimally accessible to the target users. Clinicians should be aware of these deficiencies and use shared decision making to aid patients in finding apps which are accurately informative and without harmful biases or exclusion. Four applications were ultimately found to be acceptable by the criteria of this review, but application content is constantly evolving. While providers may endorse the products highlighted in this review to their patients, general recommendation of apps developed by health systems (e.g. "Circle by Swedish") may be the most appropriate solution to ensuring quality information delivery to patients amidst a dynamic peripartum app landscape.

\section{Abbreviations}

ACOG: American College of Obstetricians and Gynecologists

CDC: Center for Disease Control and Prevention

Page 10/19 
MARS: Mobile Application Rating Scale

PRISMA: Preferred Reporting Items for Systematic Reviews and Meta-Analyses

\section{Declarations}

\section{Ethics Approval}

Not applicable. This study was reviewed by the University of Pittsburgh Institutional Review Board. It was evaluated to have no involvement of human subjects, according to the federal regulations [45 CFR 46.102(e)] and therefore given No Human Subject approval. IRB\# 2003002.

\section{Consent for Publication}

Not applicable.

\section{Availability of Data and Materials}

The datasets used and analyzed during the current study are available on Open Science Framework.

\section{Competing Interests}

TK is a co-founder of Naima Health LLC, which develops digital health tools to engage patients in clinical care.

\section{Funding}

This work was supported by TK's Institutional K-award (NIH KL2 TR001856).

\section{Authors' contributions}

LT created research protocol, conducted literature search, conducted app search and screening, evaluated apps based on research protocol, and analyzed and interpreted results.

ACV evaluated apps based on research protocol and analyzed and interpreted results.

TK created research protocol, conducted literature search, and analyzed and interpreted results.

All authors read and approved the final manuscript.

\section{Acknowledgements}

We gratefully acknowledge the research assistance of Rebecca Georgiadis, Meghana Akula, Gem Gonzales, and Kelly Nestman.

\section{References}


1. Hoyert DL, Uddin SF, Miniño AM. Evaluation of the pregnancy status checkbox on the identification of maternal deaths.

2. Creanga AA, Syverson C, Seed K, Callaghan WM. Pregnancy-related mortality in the United States, 2011-2013. Obstetrics and gynecology. 2017 Aug;130(2):366.

3. Wisner KL, Sit DK, McShea MC, Rizzo DM, Zoretich RA, Hughes CL, Eng HF, Luther JF, Wisniewski SR, Costantino ML, Confer AL. Onset timing, thoughts of self-harm, and diagnoses in postpartum women with screen-positive depression findings. JAMA psychiatry. 2013 May 1;70(5):490-8.

4. Alhusen JL, Ray E, Sharps P, Bullock L. Intimate partner violence during pregnancy: maternal and neonatal outcomes. Journal of women's health. 2015 Jan 1;24(1):100-6.

5. Creanga AA, Berg CJ, Ko JY, Farr SL, Tong VT, Bruce FC, Callaghan WM. Maternal mortality and morbidity in the United States: where are we now? Journal of women's health. 2014 Jan 1;23(1):3-9.

6. Howell EA, Egorova N, Balbierz A, Zeitlin J, Hebert PL. Black-white differences in severe maternal morbidity and site of care. American journal of obstetrics and gynecology. 2016 Jan 1;214(1):122-e1.

7. Vilda D, Wallace M, Dyer L, Harville E, Theall K. Income inequality and racial disparities in pregnancyrelated mortality in the US. SSM-population health. 2019 Dec 1;9:100477.

8. Petersen EE, Davis NL, Goodman D, Cox S, Mayes N, Johnston E, Syverson C, Seed K, ShapiroMendoza CK, Callaghan WM, Barfield W. Vital signs: pregnancy-related deaths, United States, 20112015, and strategies for prevention, 13 states, 2013-2017. Morbidity and Mortality Weekly Report. 2019 May 10;68(18):423.

9. Petersen EE, Davis NL, Goodman D, Cox S, Syverson C, Seed K, Shapiro-Mendoza C, Callaghan WM, Barfield W. Racial/ethnic disparities in pregnancy-related deaths-United States, 2007-2016. Morbidity and Mortality Weekly Report. 2019 Sep 6;68(35):762.

10. Sharps PW, Laughon K, Giangrande SK. Intimate partner violence and the childbearing year: maternal and infant health consequences. Trauma, Violence, \& Abuse. 2007 Apr;8(2):105-16.

11. Thomas JL, Lewis JB, Martinez I, Cunningham SD, Siddique M, Tobin JN, Ickovics JR. Associations between intimate partner violence profiles and mental health among low-income, urban pregnant adolescents. BMC pregnancy and childbirth. 2019 Dec 1;19(1):120.

12. Cheng D, Horon IL. Intimate-partner homicide among pregnant and postpartum women. Obstetrics \& Gynecology. 2010 Jun 1;115(6):1181-6.

13. National Research Council (US) Committee on Population; Reed HE, Koblinsky MA, Mosley WH, editors. The Consequences of Maternal Morbidity and Maternal Mortality: Report of a Workshop. Washington (DC): National Academies Press (US); 2000. APPENDIX A, Definitions.

14. Hunter LP, Rychnovsky JD, Yount SM. A selective review of maternal sleep characteristics in the postpartum period. Journal of Obstetric, Gynecologic \& Neonatal Nursing. 2009 Jan 1;38(1):60-8.

15. Wilson N, Lee JJ, Bei B. Postpartum fatigue and depression: a systematic review and meta-analysis. Journal of Affective Disorders. 2019 Mar 1;246:224-33. 
16. Hilfiker-Kleiner D, Haghikia A, Nonhoff J, Bauersachs J. Peripartum cardiomyopathy: current management and future perspectives. European heart journal. 2015 May 7;36(18):1090-7.

17. Building U.S. Capacity to Review and Prevent Maternal Deaths. (2018). Report from nine maternal mortality review committees. Retrieved from http://reviewtoaction.org/Report_from_Nine_MMRCs

18. ACOG Committee Opinion No. 736: Optimizing Postpartum Care. Obstet Gynecol. 2018;131(5):e140e150. doi:10.1097/AOG.0000000000002633.

19. Tripp N, Hainey K, Liu A, Poulton A, Peek M, Kim J, Nanan R. An emerging model of maternity care: smartphone, midwife, doctor?. Women and Birth. 2014 Mar 1;27(1):64-7.

20. Hughson JA, Daly JO, Woodward-Kron R, Hajek J, Story D. The rise of pregnancy apps and the implications for culturally and linguistically diverse women: narrative review. JMIR mHealth and uHealth. 2018;6(11):e189.

21. Liu SY, Fiorentini C, Bailey Z, Huynh M, McVeigh K, Kaplan D. Structural Racism and Severe Maternal Morbidity in New York State. Clinical Medicine Insights: Women's Health. 2019 Jun;12:1179562X19854778.

22. ACOG Committee Opinion No. 586: Health disparities in rural women. Obstet Gynecol. 2014;123(2 Pt 1):384-388. doi:10.1097/01.AOG.0000443278.06393.d6

23. Pew Research Center. 2019 Jun 12. Who owns cellphones and smartphones.

24. Perrin A. Pew Research Center. 2019 Aug 20. Smartphones help Blacks, Hispanics bridge some - but not all - digital gaps with Whites.

25. Anderson-Lewis C, Darville G, Mercado RE, Howell S, Di Maggio S. mHealth technology use and implications in historically underserved and minority populations in the United States: systematic literature review. JMIR mHealth and uHealth. 2018;6(6):e128.

26. Breton ER, Fuemmeler BF, Abroms LC. Weight loss-there is an app for that! But does it adhere to evidence-informed practices?. Translational behavioral medicine. 2011 Dec 1;1(4):523-9.

27. West JH, Hall PC, Hanson CL, Barnes MD, Giraud-Carrier C, Barrett J. There's an app for that: content analysis of paid health and fitness apps. Journal of medical Internet research. 2012;14(3):e72.

28. Thomas GM, Lupton D. Threats and thrills: pregnancy apps, risk and consumption. Health, Risk \& Society. 2016 Feb 1;17(7-8):495-509.

29. Overdijkink SB, Velu AV, Rosman AN, Van Beukering MD, Kok M, Steegers-Theunissen RP. The usability and effectiveness of mobile health technology-based lifestyle and medical intervention apps supporting health care during pregnancy: systematic review. JMIR mHealth and uHealth. 2018;6(4):e109.

30. Richardson B, Dol J, Rutledge K, Monaghan J, Orovec A, Howie K, Boates T, Smit M, Campbell-Yeo M. Evaluation of mobile apps targeted to parents of infants in the neonatal intensive care unit: systematic app review. JMIR mHealth and uHealth. 2019;7(4):e11620.

31. Knitza J, Tascilar K, Messner EM, Meyer M, Vossen D, Pulla A, Bosch P, Kittler J, Kleyer A, Sewerin P, Mucke J. German mobile Apps in rheumatology: review and analysis using the mobile application 
rating scale (MARs). JMIR mHealth and uHealth. 2019;7(8):e14991.

32. Creber RM, Maurer MS, Reading M, Hiraldo G, Hickey KT, Iribarren S. Review and analysis of existing mobile phone apps to support heart failure symptom monitoring and self-care management using the Mobile Application Rating Scale (MARS). JMIR mHealth and uHealth. 2016;4(2):e74.

33. Moher D, Liberati A, Tetzlaff J, Altman DG, Prisma Group. Preferred reporting items for systematic reviews and meta-analyses: the PRISMA statement. PLoS med. 2009 Jul 21;6(7):e1000097.

34. Centers for Disease Control and Prevention. After the Baby Arrives. https://www.cdc.gov/pregnancy/after.html. Accessed August 1, 2019.

35. S. Census Bureau (2019). U.S. Census Bureau QuickFacts. American Community Survey 5-year estimates. Retrieved from https://www.census.gov/quickfacts/fact/table/US/PST045219.

36. Stoyanov SR, Hides L, Kavanagh DJ, Zelenko O, Tjondronegoro D, Mani M. Mobile app rating scale: a new tool for assessing the quality of health mobile apps. JMIR mHealth and uHealth. 2015;3(1):e27.

37. Duckett L, Henly SJ, Garvis M, Weiss JR, Humenick SS. Predicting breast-feeding duration during the postpartum hospitalization. Western Journal of Nursing Research. 1993 Apr;15(2):177-98.

38. Wagner EA, Chantry CJ, Dewey KG, Nommsen-Rivers LA. Breastfeeding concerns at 3 and 7 days postpartum and feeding status at 2 months. Pediatrics. 2013 Oct 1;132(4):e865-75.

39. Tully KP, Stuebe AM, Verbiest SB. The fourth trimester: a critical transition period with unmet maternal health needs. American Journal of Obstetrics and Gynecology. 2017 Jul 1;217(1):37-41.

40. Kim TH, Connolly JA, Tamim H. The effect of social support around pregnancy on postpartum depression among Canadian teen mothers and adult mothers in the maternity experiences survey. BMC pregnancy and childbirth. 2014 Dec 1;14(1):162.

41. Evans M, Donelle L, Hume-Loveland L. Social support and online postpartum depression discussion groups: A content analysis. Patient education and counseling. 2012 Jun 1;87(3):405-10.

42. Cuijpers P, Brännmark JG, van Straten A. Psychological treatment of postpartum depression: a metaanalysis. Journal of clinical psychology. 2008 Jan;64(1):103-18.

43. Malat J, Hamilton MA. Preference for same-race health care providers and perceptions of interpersonal discrimination in health care. Journal of Health and Social Behavior. 2006 Jun;47(2):173-87.

44. Chen FM, Fryer GE, Phillips RL, Wilson E, Pathman DE. Patients' beliefs about racism, preferences for physician race, and satisfaction with care. The Annals of Family Medicine. 2005 Mar 1;3(2):138-43.

45. Prather C, Fuller TR, Jeffries IV WL, Marshall KJ, Howell AV, Belyue-Umole A, King W. Racism, African American women, and their sexual and reproductive health: a review of historical and contemporary evidence and implications for health equity. Health Equity. 2018 Sep 1;2(1):249-59.

46. Fleming R, Berkowitz $B$, Cheadle AD. Increasing minority representation in the health professions. The Journal of school nursing. 2005 Feb;21(1):31-9.

47. Gu J, Xu YC, Xu H, Zhang C, Ling H. Privacy concerns for mobile app download: An elaboration likelihood model perspective. Decision Support Systems. 2017 Feb 1;94:19-28. 
48. Zapata BC, Fernández-Alemán JL, Idri A, Toval A. Empirical studies on usability of mHealth apps: a systematic literature review. Journal of medical systems. 2015 Feb 1;39(2):1.

49. Carter-Edwards L, Østbye T, Bastian LA, Yarnall KS, Krause KM. Barriers to adopting a healthy lifestyle: insight from postpartum women. BMC Research Notes. 2009 Dec;2(1):1-4.

50. Vartanian K, Rinaldi JB, Pieper K, Jellison T. Effect of a Health System Sponsored Mobile App on Perinatal Health Behaviors [13E]. Obstetrics \& Gynecology. 2020 May 1;135:52S-3S.

\section{Tables}

Table 1: Coding scheme for maternal clinical risk information.

\begin{tabular}{|lll|}
\hline Maternal Health Information & & \\
\hline $\begin{array}{l}\text { Peripartum Behaviors } \\
\text { Health }\end{array}$ & Peripartum Outpatient Care & Peripartum Acute Health Risks \\
\hline Diet/Weight Trajectory & Contraceptive Options & Amniotic Fluid Embolism \\
\hline Family Planning & Depression & Anesthesia Complications \\
\hline Infant Safe Sleep & Hypertension & Cardiovascular/Heart Disease \\
\hline Postpartum Infant Care & Intimate Partner Violence & $\begin{array}{l}\text { Cerebrovascular } \\
\text { Pccident/Stroke }\end{array}$ \\
\hline Postpartum Weight Loss & Medication Use During Pregnancy & C-Section Complications \\
\hline Sexual Activity & $\begin{array}{l}\text { Medication Use During } \\
\text { Breastfeeding }\end{array}$ & Infections/Sepsis \\
\hline Sleep Quality & Postpartum Mental Health & Pulmonary Embolism \\
\hline Smoking & Postpartum Physical Health & Vaginal Birth Complications \\
\hline & Postpartum Physical/Pelvic Exam & \\
\hline & Substance Use Disorder & \\
\hline & Transitioning to Primary Care & \\
\hline
\end{tabular}

\section{Figures}




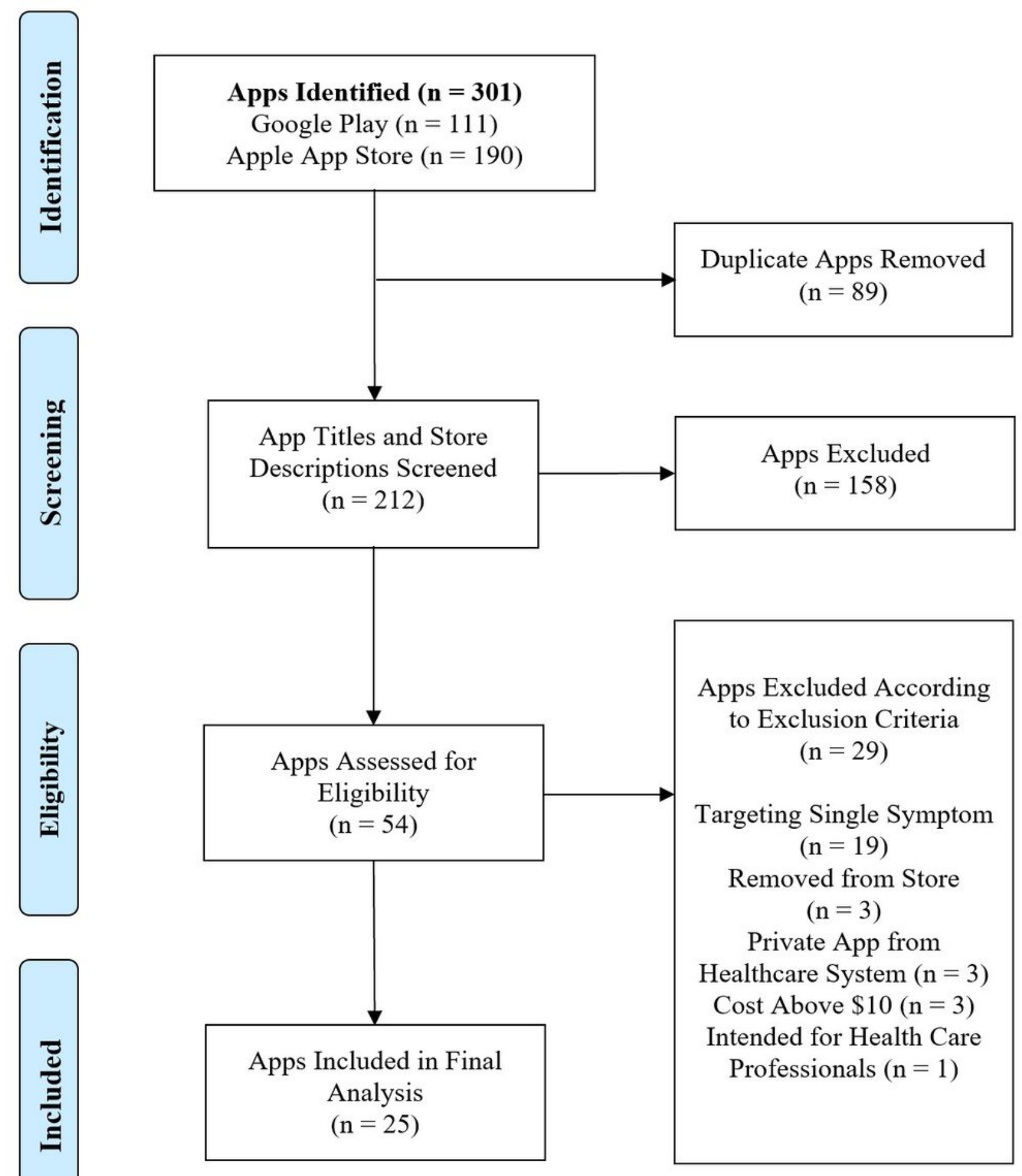

Figure 1

Preferred Reporting Items for Systematic Reviews and Meta-Analyses flowchart of search process for educational mHealth apps with postpartum maternal morbidity and mortality risk content. 


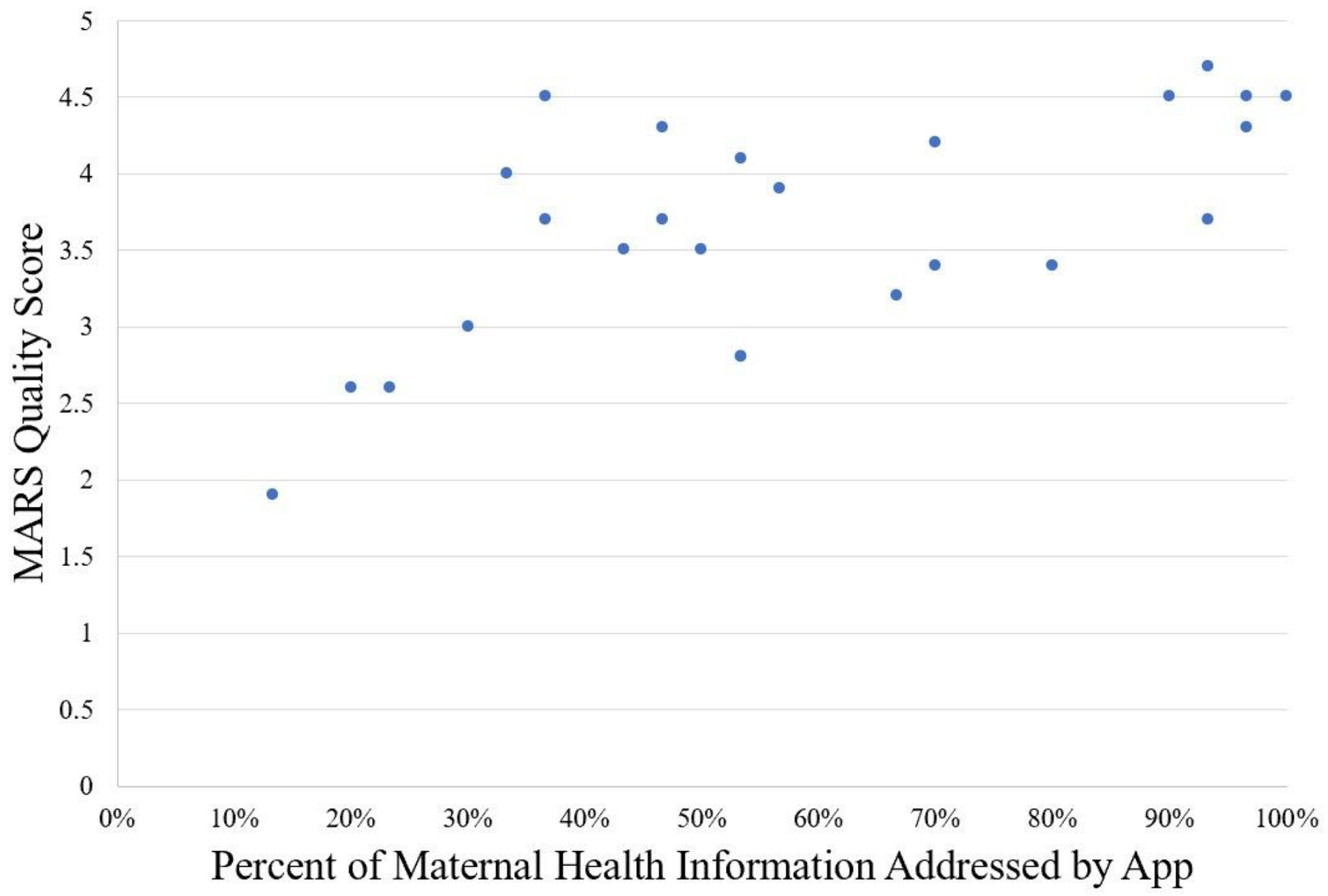

Figure 2

MARS overall quality score vs percent of maternal health information addressed by app. 


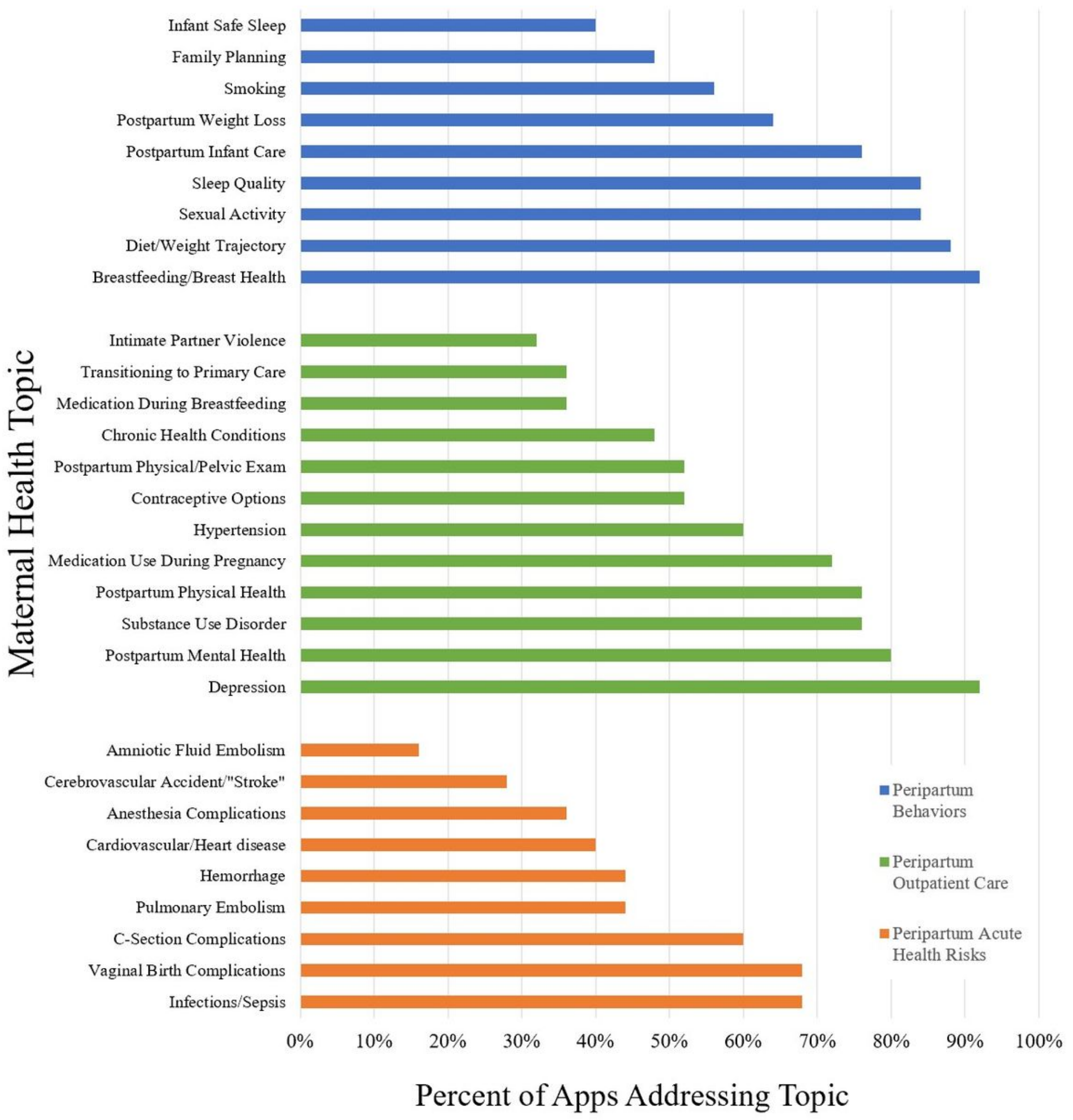

\section{Figure 3}

Percent of mobile applications addressing elements of peripartum behaviors, peripartum outpatient care, and peripartum acute health risks. 


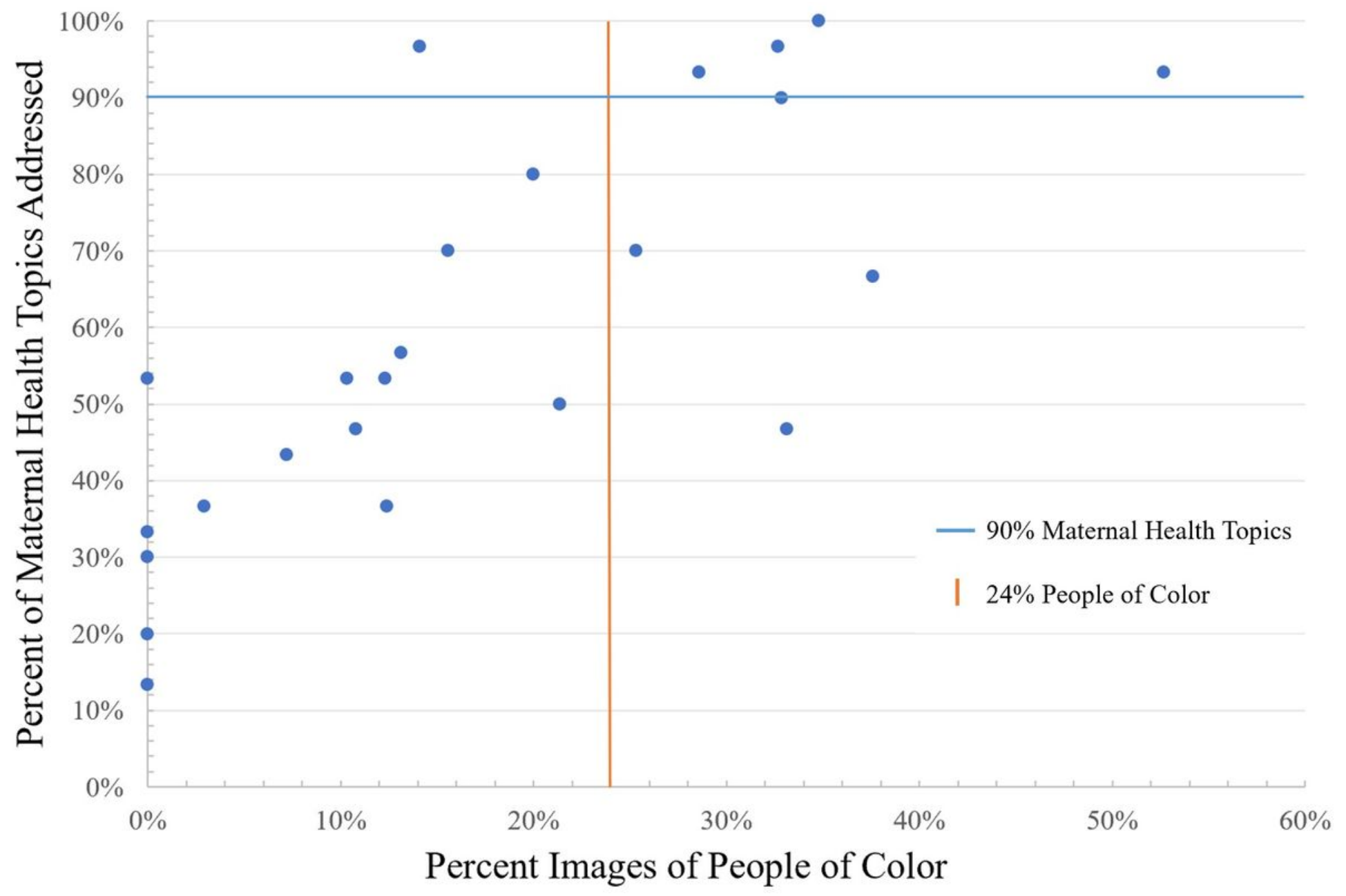

Figure 4

\section{Supplementary Files}

This is a list of supplementary files associated with this preprint. Click to download.

- SupplementalAppendixA.docx 